\title{
$625 \mathrm{~kb}$ microduplication at Xp22.12 including RPS6KA3 in a child with mild intellectual disability
}

\author{
Veronica Bertini ${ }^{1}$, Francesca Cambi ${ }^{1}$, Rossella Bruno ${ }^{1}$, Benedetta Toschi ${ }^{1}$, Francesca Forli ${ }^{2}$, Stefano Berrettini ${ }^{2}$, \\ Paolo Simi ${ }^{1}$ and Angelo Valetto ${ }^{1}$
}

Here, we report on a patient with a $625 \mathrm{~kb}$ duplication in Xp22.12, detected by array comparative genomic hybridization (CGH). The duplicated region contains only one gene, RPS6KA3, that results in partial duplication. The same duplication was present in his mother and his maternal uncle. This partial duplication inhibits the RPS6KA3 expression, mimicking the effect of loss-offunction mutations associated with Coffin-Lowry syndrome (CLS). The phenotype of the patient here presented is not fully evocative of this syndrome because he does not present some of the facial, digital and skeletal abnormalities that are considered the main diagnostic features of CLS. This case is one of the few examples where RPS6KA3 mutations are associated with a non-specific X-linked mental retardation.

Journal of Human Genetics (2015) 60, 777-780; doi:10.1038/jhg.2015.106; published online 10 September 2015

Here, we report on a new case, having a duplication in Xp22.12 of about $625 \mathrm{~kb}$, detected by array comparative genomic hybridization (CGH); this region contains only one gene, RPS6KA3, that results in partial duplication. This duplication was inherited from his mother and is present also in his maternal uncle.

The patient is a 12-year-old boy, the only child of unrelated parents (Figure 1). At delivery, the mother and father were 26 and 33 years old, respectively. The proband was born at term, after an uneventful pregnancy. Birth weight was $2880 \mathrm{~g}(\sim 15$ th centile), length $47 \mathrm{~cm}$ (3rd-15th centile) and head circumference $34.2 \mathrm{~cm}$ ( $<50$ th centile). The mother had four miscarriages and a therapeutic abortion for fetal lung malformation. Family history was unremarkable, except for an uncle (the mother's brother) who was reported to have had learning difficulties, a mild intellectual disability (ID), a facial aspect similar to his nephew and obesity.

Shortly after birth, the patient had a brief cyanotic episode that did not require supported ventilation. His developmental milestones were mildly delayed: he sat without aid at 10 months and walked with support by 16 months. Speech was delayed, especially in the expressive area, and he received speech therapy from the age of 4 years. He attended school where a mild ID was noted and he was provided with a support teacher.

Routine blood, plasma and urine investigations were in the normal range; thyroid function tests and neurometabolic screenings were normal. Ultrasound of abdomen and heart were normal, as was an ophthalmologic evaluation. At 9 years of age, he was diagnosed with moderate bilateral sensorineural hearing loss that required the use of a hearing aid. Cytomegalovirus test was negative.

Sleeping and awake electroencephalogram records showed significant paroxysmal abnormalities (spikes, polispikes and sharp-wave complexes) in the left centrotemporal region, sometimes extending to the same region on the right. Brain magnetic resonance imaging showed several bilateral focal hyperintense areas, especially in the subcortical frontoparietal white matter and around the trigones, likely due to gliosis. He never exhibited clinical seizures.

The patient came to our attention at the age of 11 years because of language difficulties, hearing loss and mild ID. Height was $136.5 \mathrm{~cm}$ ( $\sim 15$ th centile), weight $37.5 \mathrm{~kg}$ ( $>50$ th centile) and head circumference $55.5 \mathrm{~cm}$ (90th centile). He showed a flat facial profile with high forehead, a short nose with anteverted nares, small, low-set, posteriorly rotated ears, a high palate and dental crowding (Figure 1). He was tested for intelligence quotient, which results at the lower limit $(\mathrm{QIG}=70)$, with verbal capacities $(\mathrm{QIV}=65)$ lower than performance abilities (QIP = 78).

After obtaining written informed consent for genetic testing, standard chromosomal analysis (450 band resolution) was performed in the proband and resulted normal. Molecular analysis of GJB2 and GJB6 genes and the A1555G mitochondrial mutation were normal. Array CGH, using a $44 \mathrm{~K}$ oligo platform (Agilent Technologies, Santa Clara, CA, USA) with an average probe spatial resolution of about $35 \mathrm{~kb}$, detected an interstitial duplication in Xp22.12. To better define the duplication extent, we used a higher density array $(4 \times 180)$, with an average spatial resolution of about $20 \mathrm{~kb}$. The duplication extent is $625 \mathrm{~kb}$, starting at position 20208171 and ending at 20833589 (hg19 map) (Figure 2a). The distal non-duplicated oligo maps in position 20204496 , and the proximal non-duplicated oligo maps in 20 847715 . The duplicated region contains only one gene, RPS6KA3, that results in partial duplication.

According to the database http://www.ncbi.nlm.nih.gov/gene (ENST00000379565, hg 19 map), exons 1-7 are duplicated, exons

${ }^{1}$ Laboratorio di Genetica Medica, A.O.U. Pisana, Ospedale S. Chiara, Pisa, Italy and 'Unita' di Audiologia e Foniatria Universitaria, A.O.U. Pisana, Ospedale S. Chiara, Pisa, Italy Correspondence: Dr A Valetto, Medical Genetic Unit, A.O.U. Pisana, Ospedale S. Chiara, via Roma 57, 56100 Pisa, Italy. E-mail: a.valetto@ao-pisa.toscana.it

Received 3 April 2014; revised 9 July 2015; accepted 13 July 2015; published online 10 September 2015 

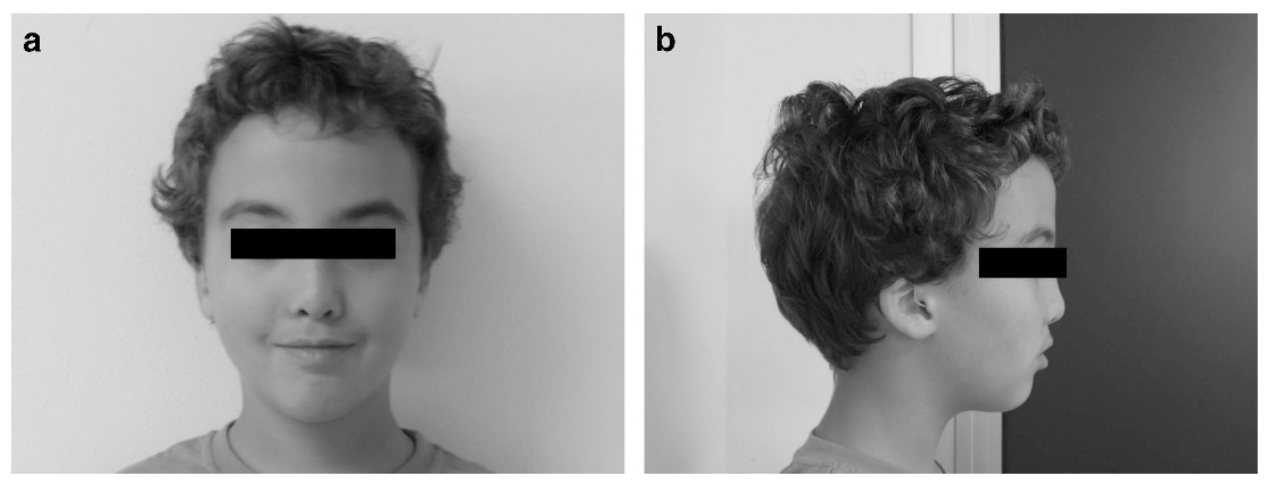

Figure 1 (a) Frontal view of the patient (b) Lateral right view of the patient. A full color version of this figure is available at the Journal of Human Genetics journal online.

8-9 fall in the region between the last non-duplicated and the first duplicated oligo, and exons 10-22 are not duplicated (Figure 2b).

Array CGH analysis was extended to his relatives and showed the same duplication in his mother and in his maternal uncle. X-chromosome inactivation pattern in the mother was determined by the HUMARA (human androgen receptor) assay; ${ }^{1}$ results were compatible with a random inactivation.

To confirm this partial duplication, we performed a copy number assay on DNA of the proband, his mother and his maternal uncle, using two probes: RPS6KA3 Hs05693240_cn, which maps in the duplicated region (intron 2), and RPS6KA3 Hs02029033_cn, which maps within the non-duplicated region (exon 21) (Life Technologies, New York, NY, USA). As can be seen in Figure 2c, this test confirmed the array CGH results: when analyzed with the first probe, the patient, his mother and his uncle showed an additional copy compared with normal controls.

To verify whether the duplication was in tandem, we performed a fluorescence in situ hybridization using a commercial probe RP11367L17 (BlueGnome, Cambridge, UK), containing the RPS6KA3 gene. There was no ectopic insertion because the probe gave on metaphase spreads of the proband only one signal on Xp chromosome, whereas in his mother, a signal for each X chromosome was present (one signal always more intense than the other), according to an in tandem duplication (Figures $2 \mathrm{~d}$ and $\mathrm{e}$ ).

To assess the effect of the duplication on the transcription, we analyzed the expression profile of RPS6KA3 by quantitative real timePCR (qRT-PCR).

Total RNA was extracted from peripheral blood cells of the proband, his mother and healthy controls using the mirVana Paris kit (Life Technologies). No RNA sample was available from the maternal uncle. RNA was reverse-transcripted through the High capacity cDNA reverse transcription kit (Life Technologies). qRTPCR was performed using commercial predesigned Taqman probes mapping in the non-duplicated region: RPS6KA3 Hs01078601_g1 (located in exon 21-22 boundary) and RPS6KA3 Hs00177936_m1 (located in exon 17-18 boundary). GAPDH (Hs02786624_g1) was used for normalization (Life Technologies). To calculate the relative changes in gene expression, we applied the 2-DDct method. ${ }^{2-3}$ The RPSK6A3 transcript in the proband, compared with the healthy controls, showed a decreased expression in both cases (Figure 2f). His healthy mother also shows a low (even if higher than her son) expression of RPS6KA3 in peripheral blood, but she does not present the clinical phenotype. This could be a consequence of the X-chromosome random inactivation, that in some tissues (that is, brain) could favor the normal $\mathrm{X}$, or maybe because other epigenetic factors may have a role in modulating the clinical variability seen in Coffin-Lowry syndrome (CLS).

RPS6KA3 encodes a serine/threonine kinase, RSK2 (ribosomal S6 kinase2). In humans, RSK2 belongs to a family that includes four members, RSK1-4, encoded by different genes, with a highly conserved structure. ${ }^{4}$ RSK2 is a key member of several pathways regulating proliferation, differentiation, cell survival, chromatin-remodeling events, neurite growth and synaptic plasticity.

In humans and in mice, RPS6KA3 is widely expressed in embryonic and adult brain; the highest levels are observed in regions with high synaptic activity, essential to cognitive function, including the neocortex, the hippocampus and the Purkinje cells. ${ }^{5-7}$

In our patient, the duplication of RPS6KA3 is partial because it involves only the exons $1-7$, that code for the $\mathrm{N}$-terminal kinase catalytic domain of the protein. This partial duplication inhibits the gene expression, as shown by qRT-PCR data, mimicking the effect of a loss-of-function mutation. The present case is similar to the one described by Pereira et al. ${ }^{8}$ They reported a $6100 \mathrm{bp}$ duplication of RPS6KA exons 17-20 in a patient with CLS; the duplication was in tandem, mediated by Alu elements that fused together to reconstruct a chimeric Alu sequence. The effect of this duplication was to dramatically inhibit gene expression, as shown by reduced protein level in lymphoblast cell lines. The functional inactivation of a gene as consequence of in tandem duplications is a well-known mechanism, described in many disease models. ${ }^{9}$

Loss-of-function mutations of RPS6KA3 are associated with CLS. In most of the male patients over 5-10 years of age, the disease has a well-defined phenotype and can easily be diagnosed by professionals trained in genetic disorders. ${ }^{10-11}$

However, in some cases, the presentation of CLS may be markedly variable in severity and in expression of clinical features, and rare isolated ID has been reported. ${ }^{12}$

The phenotype of the patient here presented is not fully evocative of CLS. He shares with CLS patients only short stature, a high narrow palate and an abnormal dental position, but he does not show the typical facial dysmorphisms of CLS such as prominent forehead, orbital hypertelorism, downward-slanting palpebral fissures, epicanthic folds, thick nasal septum, large and prominent ears, large mouth, and thick everted lips.

He neither has the digital abnormalities nor has the progressive skeletal alterations that, along with the facial anomalies, are considered the cardinal diagnostic features of this syndromes. His fingers are not tapering and stubby, and he does not manifest a delayed bone development, spinal kyphosis/scoliosis, and pectus carinatum or excavatum, that are often reported in CLS. 
a

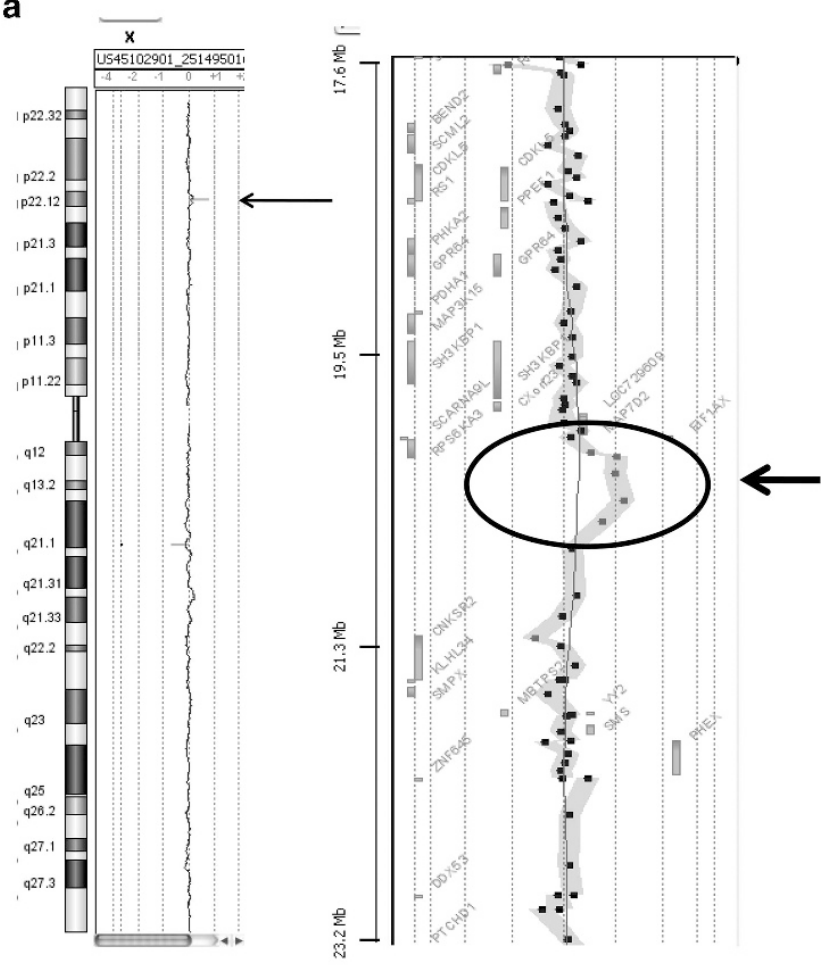

d

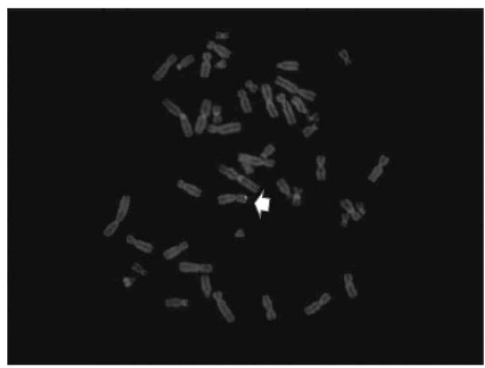

e

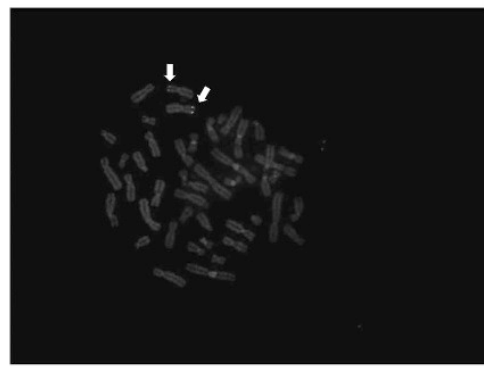

b

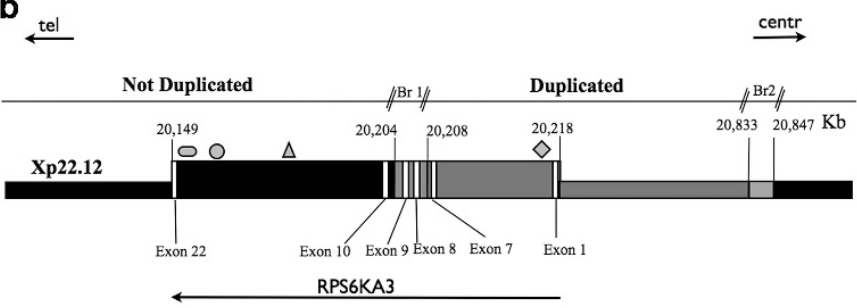

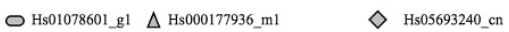

O Hs02029033_cn

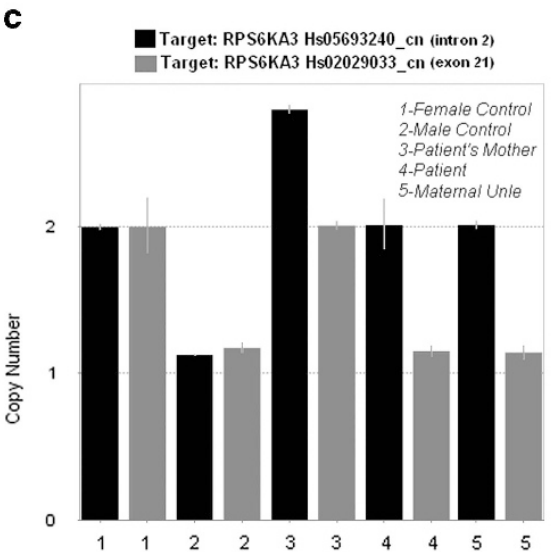

f

Target RPS6KA3: Hs_01078601_g1

Target RPS6KA3: Hs_00177936_m1

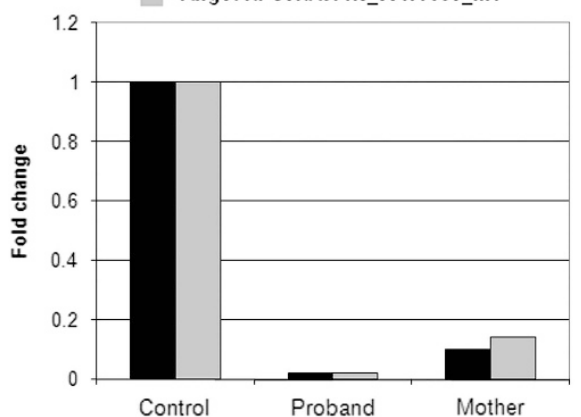

Figure 2 (a) Array CGH profile of the chromosome $X$ on the left side. On the right side, the anomaly is highlighted (circle). (b) Schematic representation of the region Xp22.12. Red bar represents the duplicated region, starting from position 20208 to $20218 \mathrm{~kb}$ (hg19 map). The black bar represents the nonduplicated region. The grey bars refer to the interval between duplicated and non-duplicated oligos. The position of the gene RPS6KA3 is given, with indication of the exons interested by the duplication. $\mathrm{Br}$ is breakpoint; centr is centromere; tel is telomere. Probes used in the validation of array CGH data are shown (diamond and circle), and in the qRT-PCR (rectangle and triangle). (c) Panel showing the copy number assay results. Black bars refer to probe Hs05693240 cn (mapping in intron 2), showing a duplication in the proband (4), his mother (3) and his uncle (5), whereas grey bars refer to probe Hs2029033_cn, (mapping in exon 21, non duplicated), showing results similar to normal controls (1 and 2). (d) Fluorescence in situ hybridization (FISH) using the probe RP11-367L17 on metaphase spreads of the proband, where no ectopic signals are detected. (e) FISH using the probe RP11-367L17 on metaphase spreads of the proband's mother, where one signal on each X chromosome is detected. A signal on an X chromosome is stronger than the other one, suggestive of an in tandem duplication. (f) qRT-PCR results showed a marked reduced expression of the gene RPS6KA3 with both probes Hs01078601_g1 (black bars) and Hs00177936 (grey bars) in the proband and in his mother. A full color version of this figure is available at the Journal of Human Genetics journal online.

On the other hand, his neurological features are typical of CLS. He shares with these patients a mild ID, a mild psycho-motor retardation, a speech delay and a sensorineural deafness, aspect that is reported only in about $30 \%$ of cases. However, all these clinical features are often encountered in subjects with chromosomal imbalances, and then are not in themselves evocative of CLS.

As the best of our knowledge, only five families with mutations in RPS6KA3 and an atypical phenotype not reminiscent of CLS have been documented; ${ }^{12-14}$ it is worth noting that these cases have been identified by screening for RSK2 mutations of families with undiagnosed non-syndromic X-linked mental retardation.

These data suggest that RPS6KA3 mutations not producing the classical CLS phenotype are a rare, but not insignificant, cause of nonsyndromic X-linked mental retardation. The prevalence of CLS is probably underestimated, as a strict reliance on the typical dysmorphic features may result in a missed diagnosis. The use of molecular diagnostic technology, such as array CGH, represents a powerful tool to discover the molecular basis of those pathological phenotypes not 
reminiscent of a well-defined syndrome. The direct consequence is a redefinition of the diagnostic criteria of a syndrome: including new features, and often removing features previously believed to be necessary, but present only in a subset of patients.

\section{CONFLICT OF INTEREST}

The authors declare no conflict of interest.

1 Allen, R. C., Zoghbi, H. Y., Moseley, A. B., Rosenblatt, H. M. \& Belmont, J. W. Methylation of Hpall and Hhal sites near the polymorphic CAG repeat in the human androgen-receptor gene correlates with X chromosome inactivation. Am. J. Hum. Genet. 51, 1229-1239 (1992)

2 Livak, K. J. \& Schmittgen, T. D. Analysis of relative gene expression data using real time quantitative PCR and the $2^{\wedge}$ [-delta delta C(T)] Method. Methods 25, 402-408 (2001).

3 Schmittgen, T. D. \& Livak, K. J. Analyzing real-time PCR data by the comparative CTmethod. Nat. Protoc. 3, 1101-1108 (2008)

4 Hauge, C. \& Frödin, M. RSK and MSK in MAP kinase signalling. J. Cell. Sci. 119, 3021-3023 (2006)

5 Darcq, E., Koebel, P., Del Boca, C., Pannetier, S., Kirstetter, A. S., Garnier, J. M. et al. RSK2 signaling in brain habenula contributes to place aversion learning. Learn. Mem. 18, 574-578 (2011).
6 Poirier, R., Jacquot, S., Vaillend, C., Soutthiphong, A. A., Libbey, M., Davis, S. et al Deletion of the Coffin-Lowry syndrome gene Rsk2 in mice is associated with impaired spatial learning and reduced control of exploratory behavior. Behav. Genet. 37 31-50 (2007).

7 Mehmood, T., Schneider, A., Sibille, J., Marques Pereira, P., Pannetier, S., Ammar, M. R. et al. Transcriptome profile reveals AMPA receptor dysfunction in the hippocampus of the Rsk2-knockout mice, an animal model of Coffin-Lowry syndrome. Hum. Genet. 129, 9255-9269 (2011)

8 Pereira, P. M., Heron, D. \& Hanauer, A. The first large duplication of the RSK2 gene identified in a Coffin-Lowry syndrome patient. Hum. Genet. 122, 541-543 (2007).

9 Thienpont, B., de Ravel, T., Van Esch, H., Van Schoubroeck, D., Moerman, P., Vermeesch, J. R. et al. Partial duplication of the ATRX gene cause the ATR-X syndrome. Eur. J. Hum. Genet. 15, 1094-1097 (2007).

10 Hanauer, A. \& Young, I. D. Coffin-Lowry syndrome clinical and molecular features. J. Med. Genet. 39, 705-713 (2002).

11 Pereira, P. M., Schneider, A., Pannetier, S. \& Heron, D. Coffin-Lowry syndrome. Eur. J. Hum. Genet. 18, 627-633 (2010).

12 Manouvrier-Hanu, S., Amiel, J., Jacquot, S., Merienne, K., Moerman, A., Coëslier, A. et al. Unreported RSK2 missense mutation in two male sibs with an unusually mild form of Coffin-Lowry Syndrome. J. Med. Genet. 36, 775-778 (1999).

13 Merienne, K., Jacquot, S., Pannetier, S., Zeniou, M., Bankier, A., Gecz, J. et al A missense mutation in RPS6KA3 (RSK2) responsible for non-specific mental retardation. Nat. Genet. 22, 13-14 (1999).

14 Field, M., Tarpey, P., Boyle, J., Edkins, S., Goodship, J., Luo, Y. et al. Mutations in the RSK2(RPS6KA3) gene cause Coffin-Lowry syndrome and nonsyndromic X-linked mental retardation. Clin. Genet. 70, 509-515 (2006). 